\title{
Correction to: Relation between myocardial blood flow and cardiac events in diabetic patients with suspected coronary artery disease and normal myocardial perfusion imaging
}

Roberta Assante, $\mathrm{MD}, \mathrm{PhD},{ }^{\mathrm{a}}$ Ciro Gabriele Mainolfi, MD, ${ }^{\mathrm{a}}$ Emilia Zampella, MD, $\mathrm{PhD},{ }^{a}$ Valeria Gaudieri, MD, PhD, ${ }^{a}$ Carmela Nappi, MD, PhD, ${ }^{a}$ Teresa Mannarino, $M D,{ }^{a}$ Adriana D'Antonio, MD, ${ }^{a}$ Parthiban Arumugam, MD, ${ }^{b}$ Mario Petretta, MD, FAHA, ${ }^{c}$ Alberto Cuocolo, MD, a and Wanda Acampa, MD, PhD ${ }^{\mathrm{a}, \mathrm{d}}$

${ }^{a}$ Department of Advanced Biomedical Sciences, University Federico II, Naples, Italy

${ }^{\mathrm{b}}$ Nuclear Medicine Center, Central Manchester University Teaching Hospitals, Manchester, UK

c Department of Translational Medical Sciences, University Federico II, Naples, Italy

${ }^{d}$ Institute of Biostructure and Bioimaging, National Council of Research, Naples, Italy

doi: $10.1007 / \mathrm{s} 12350-021-02832-2$

\section{CORRECTION TO: JOURNAL OF NUCLEAR CARDIOLOGY VOLUME 28, NUMBER 4; 1222- 33 HTTPS://DOI.ORG/10.1007/S12350-021 - 02533-W}

Please note that an incorrect Chinese abstract was initially attached to this article. The corrected Chinese abstract is below:

摘要

背景: 我们在糖尿病可疑冠心病 (CAD) 需行PET/ CT检查的患者中, 评估了心脏结构异常和冠状动脉 舒张功能的预后价值。

方法: 分析了无明显的CAD并正常心肌灌注的 451 位糖尿病患者和 451 位非糖尿病患者。从动态的 静息和负荷成像中计算得出心肌血流量 (MBF) 。 心 肌血流储备 (MFR) 定义为充血与基础MBF的比例, 当 $<2$ 时被认为减少。
结果: 在平均 44 个月的随访期间内共发生了 33 起 事件。糖尿病患者的年事件发生率 (AER) 高于非糖 尿病患者 $(1.4 \%$ vs. $0.3 \%, \mathrm{P}<.001)$ 。 MFR降低的糖 尿病患者的AER高于MFR保留的糖尿病患者 (3.3\% vs. $0.4 \%, \mathrm{P}<.001)$ 。Cox分析表明, 年龄, BMI和 MFR降低是糖尿病患者中事件发生的独立预测因 子。与非糖尿MFR $<2$ 的患者相比, MFR降低的糖尿 病患者的无事件发生的生存率更低 $(\mathrm{P}<.001) \circ \mathrm{MFR}$ 正常的糖尿病患者与MFR降低无糖尿病的患者的无 事件发生的生存率相似。

结论: $\square$ MFR保留的非糖尿病患者相比, MFR降 低的糖尿病患者的AER较高, 无事件发生的生存率较 低。

JNC thanks Zhuo He, B.S., Michigan Technological University, Min Zhao, M.D., Ph.D., Xiangya Hospital, and Weihua Zhou, Ph.D., Michigan Technological University, for providing the translated abstract.

Publisher's Note Springer Nature remains neutral with regard to jurisdictional claims in published maps and institutional affiliations.
The original article can be found online at https://doi.org/10.1007/ s12350-021-02533-w.

Reprint requests: Wanda Acampa, MD, PhD, Department of Advanced Biomedical Sciences, University Federico II, Naples, Italy; acampa@unina.it

J Nucl Cardiol 2022;29:892.

$1071-3581 / \$ 34.00$

Copyright (C) 2021 American Society of Nuclear Cardiology. 